\title{
On discrete-domain multidimensional sinusoidal models
}

\author{
DEBASIS KUNDU* ${ }^{*}$ and SWAGATA NANDI $\ddagger$ \\ $\dagger$ Department of Mathematics and Statistics, Indian Institute of Technology, Kanpur 208016, India \\ $¥$ Statistics and Mathematics Division, Indian Statistical Institute, 7 SJS Sansanwal Marg, \\ New Delhi 110016, India
}

(Received 29 March 2005; in final form 5 November 2005)

\begin{abstract}
We consider three-dimensional sinusoidal frequency model in a random field. Three-dimensional frequency model has wide applications in statistical signal processing. In this article, we mainly consider the usual least squares estimators and the estimators that can be obtained by maximizing the periodogram function. We obtain consistency and asymptotic normality property of both the estimators. It is observed that they are asymptotically equivalent. Finally we generalize the results to the multidimensional case.
\end{abstract}

Keywords: Multidimensional sinusoidal signals; Least squares estimators; Strong consistency

AMS Subject Classification: 62F12;62M10

\section{Introduction}

In this paper, we consider the following three-dimensional (3-D) frequency model:

$$
\begin{aligned}
y\left(n_{1}, n_{2}, n_{3}\right)= & \sum_{k=1}^{p}\left[A_{k}^{0} \cos \left(n_{1} \lambda_{k 1}^{0}+n_{2} \lambda_{k 2}^{0}+n_{3} \lambda_{k 3}^{0}\right)+B_{k}^{0} \sin \left(n_{1} \lambda_{k 1}^{0}+n_{2} \lambda_{k 2}^{0}+n_{3} \lambda_{k 3}^{0}\right)\right] \\
& +X\left(n_{1}, n_{2}, n_{3}\right),
\end{aligned}
$$

for $n_{j}=1, \ldots, N_{j}, j=1,2$ and 3. Here, $A_{k}^{0}$ and $B_{k}^{0}$ are unknown real amplitudes and $\lambda_{k 1}^{0}, \lambda_{k 2}^{0}$ and $\lambda_{k 3}^{0}$ are unknown frequencies, $\lambda_{k 1}^{0}, \lambda_{k 2}^{0}, \lambda_{k 3}^{0} \in(0, \pi)$. The error random variable $X\left(n_{1}, n_{2}, n_{3}\right)$ is from a stationary random field, and it satisfies Assumption 1 (will be defined in the next section). The number of components ' $p$ ' is assumed to be known. Given a sample $\left\{y\left(n_{1}, n_{2}, n_{3}\right), n_{j}=1, \ldots, N_{j}, j=1,2\right.$ and 3$\}$, the problem is to estimate $A_{k}$ 's, $B_{k}$ 's and $\lambda_{k j}$ 's.

This is an important problem in statistical signal processing. See, for example, the site http://www.mddsp.enel.ucalgary.ca/people/bruton/enel699/chap1_2002.pdf of Professor Len Bruton, where the author has demonstrated several applications of this particular model in

*Corresponding author. Email: kundu@iitk.ac.in; Tel.: +91-512-2597141; Fax: +91-512-2597500 
signal processing. For some other applications on multidimensional (m-D) periodic signals, such as the radar imaging of scatterers, real aperture radar imaging and m-D NMR data, the readers are referred to Hua [1], Liang et al. [2] and Chylla and Markley [3].

Note that model (1) is an extension of the one-dimensional (1-D) frequency model

$$
y(n)=\sum_{k=1}^{p}\left[A_{k}^{0} \cos \left(n \lambda_{k}^{0}\right)+B_{k}^{0} \sin \left(n \lambda_{k}^{0}\right)\right]+X(n) .
$$

Here, $\{X(n)\}$ is a sequence of 1-D stationary linear process satisfying the 1-D analog of Assumption 1. For a detailed description of $X(n)$, see Assumption 1 of Kundu [4]. Note that model (2) is a very important and well-discussed model in time series and signal processing literature. Several authors, for example, Fisher [5], Hannan [6,7], Walker [8], Kundu [4, 9] and Rice and Rosenblatt [10], consider this or similar kinds of models and discuss different properties of the corresponding estimators. Stoica [11] provides an extensive list of references related to model (2) till that time.

It may also be mentioned that the 3-D model (1) is also an extension of two-dimensional (2-D) frequency model, which can be written as follows:

$$
y(m, n)=\sum_{k=1}^{p}\left[A_{k}^{0} \cos \left(m \lambda_{k}^{0}+n \mu_{k}^{0}\right)+B_{k}^{0} \sin \left(m \lambda_{k}^{0}+n \mu_{k}^{0}\right)\right]+X(m, n) .
$$

Here, $\lambda_{k}^{0}$ and $\mu_{k}^{0}$ are unknown frequencies and $\{X(m, n)\}$ is a 2-D sequence of a stationary random field. This is also a very important and well-studied model in statistical signal processing and texture analysis. Several authors consider model (3) or its variants under different assumptions on $X(m, n)$. See, for example, Barbieri and Barone [12], Cabrera and Bose [13], Chun and Bose [14], Hua [15], Kundu and Gupta [16], Lang and McClellan [17], Kundu and Mitra [18], Nandi and Kundu [19], Kundu and Nandi [20,21], Rao et al. [22] and Mitra and Stoica [23]. Estimation of different parameters, asymptotic properties of different estimators and Cramer-Rao lower bound are obtained when $X(m, n)$ 's are independent and identically distributed (i.i.d.) or when they are from a stationary linear process.

We use the following notation in this article.

$$
\begin{array}{cl}
\mathbf{n}=\left(n_{1}, n_{2}, n_{3}\right) & \mathbf{j}=\left(j_{1}, j_{2}, j_{3}\right) \\
\mathbf{n}-\mathbf{j}=\left(n_{1}-j_{1}, n_{2}-j_{2}, n_{3}-j_{3}\right) & \lambda=\left(\boldsymbol{\lambda}_{1}, \lambda_{2}, \lambda_{3}\right) \\
\lambda_{k}=\left(\lambda_{k 1}, \lambda_{k 2}, \lambda_{k 3}\right) & \lambda_{k}^{0}=\left(\lambda_{k 1}^{0}, \lambda_{k 2}^{0}, \lambda_{k 3}^{0}\right) \\
\boldsymbol{\theta}_{k}=\left(A_{k}, B_{k}, \lambda_{k 1}, \lambda_{k 2}, \lambda_{k 3}\right) & \boldsymbol{\theta}_{k}^{0}=\left(A_{k}^{0}, B_{k}^{0}, \lambda_{k 1}^{0}, \lambda_{k 2}^{0}, \lambda_{k 3}^{0}\right) \quad \text { for } k=1, \ldots, p \\
\boldsymbol{\theta}=\left(\boldsymbol{\theta}_{1}, \ldots, \boldsymbol{\theta}_{p}\right) & \boldsymbol{\theta}^{0}=\left(\boldsymbol{\theta}_{1}^{0}, \ldots, \boldsymbol{\theta}_{p}^{0}\right) \\
N=N_{1} \cdot N_{2} \cdot N_{3} & N_{(1)}=\min \left\{N_{1}, N_{2}, N_{3}\right\} \\
\sum_{\mathbf{n}=\mathbf{1}}^{N}=\sum_{n_{1}=1}^{N_{1}} \sum_{n_{2}=1}^{N_{2}} \sum_{n_{3}=1}^{N_{3}} & \sum_{\mathbf{j}=-\infty}^{\infty}=\sum_{j_{1}=-\infty}^{\infty} \sum_{j_{2}=-\infty}^{\infty} \sum_{\lambda_{3}=-\infty}^{\infty} \\
\sup _{\lambda_{1}, \lambda_{2}, \lambda_{3}} & \text { a.s. }=\text { almost sure convergence; }
\end{array}
$$




$$
\begin{aligned}
\mu(\boldsymbol{\theta}) & =\sum_{k=1}^{p}\left(A_{k} \cos \left(n_{1} \lambda_{k 1}+n_{2} \lambda_{k 2}+n_{3} \lambda_{k 3}\right)+B_{k} \sin \left(n_{1} \lambda_{k 1}+n_{2} \lambda_{k 2}+n_{3} \lambda_{k 3}\right)\right) \\
& =\sum_{k=1}^{p}\left(A_{k} \cos \left(\mathbf{n} \cdot \lambda_{k}\right)+B_{k} \sin \left(\mathbf{n} \cdot \lambda_{k}\right)\right) .
\end{aligned}
$$

The main aim of this article is to consider the 3-D frequency model and to study different properties of the estimators under the linear stationarity assumption on $X(\mathbf{n})$. The stationarity assumption we impose on $X(\mathbf{n})$ is a natural extension from 1-D or 2-D case to the 3-D case. The two estimators we propose are strongly consistent and their asymptotic distributions are also obtained. It gives an idea of the rate of convergence of the proposed estimators and they can be used to construct confidence bounds of the unknown parameters. We propose mainly two different estimators. The first one is the usual least squares estimators (LSEs), which can be obtained by minimizing

$$
\sum_{\mathbf{n}=\mathbf{1}}^{N}\left[y(\mathbf{n})-\sum_{k=1}^{p}\left(A_{k} \cos \left(\mathbf{n} \cdot \lambda_{k}\right)+B_{k} \sin \left(\mathbf{n} \cdot \lambda_{k}\right)\right)\right]^{2}
$$

with respect to the unknown parameters.

The second estimators are called the approximate least squares estimators (ALSEs) and they can be obtained by maximizing the periodogram function defined as follows:

$$
\frac{1}{N}\left|\sum_{\mathbf{n}=1}^{N} y(\mathbf{n}) \mathrm{e}^{-i(\lambda \cdot \mathbf{n})}\right|^{2} .
$$

Note that once the frequencies are estimated, the unknown parameters $A_{k}$ 's and $B_{k}$ 's can be obtained by using the simple regression technique and it will be discussed in section 3 . It is observed that the LSEs and ALSEs are asymptotically equivalent under Assumption 1. Both of them provide consistent estimators and they have the same asymptotic distribution.

The rest of the article is organized as follows. In section 2, we provide the consistency of the LSEs and also obtain their joint asymptotic distributions. The consistency and the asymptotic normality results of the ALSEs are discussed in section 3. Section 4 extends the results to the general $\mathrm{m}-\mathrm{D}$ case, and finally, we conclude the article in section 5.

\section{Consistency and asymptotic distributions of the LSE's}

In this section, we provide the consistency of the LSEs of the parameters of model (1). For brevity, first we consider $p=1$ and in that case, we will use $\lambda_{j}, \lambda_{j}^{0}, j=1,2,3, \boldsymbol{\theta}$ and $\boldsymbol{\theta}^{\mathbf{0}}$ suppressing $k$. Therefore, the LSEs of $\boldsymbol{\theta}^{0}=\left(A^{0}, B^{0}, \lambda_{1}^{0}, \lambda_{2}^{0}, \lambda_{3}^{0}\right)$ can be obtained by minimizing

$$
Q_{N}(\boldsymbol{\theta})=\sum_{\mathbf{n}=\mathbf{1}}^{N}(y(\mathbf{n})-\mu(\boldsymbol{\theta}))^{2}
$$

with respect to $\boldsymbol{\theta}=\left(A, B, \lambda_{1}, \lambda_{2}, \lambda_{3}\right)$. The LSE of $\boldsymbol{\theta}^{0}$ will be denoted by $\hat{\boldsymbol{\theta}}=$ $\left(\hat{A}, \hat{B}, \hat{\lambda}_{1}, \hat{\lambda}_{2}, \hat{\lambda}_{3}\right)$. We need the following assumptions to prove the consistency of the LSEs. 
Assumption 1 Let us denote the set of positive integers by $Z .\left\{X\left(n_{1}, n_{2}, n_{3}\right) ; n_{1}, n_{2}, n_{3} \in Z\right\}$ can be represented as follows:

$$
X(\mathbf{n})=\sum_{\mathbf{j}=-\infty}^{\infty} a\left(j_{1}, j_{2}, j_{3}\right) e\left(n_{1}-j_{1}, n_{2}-j_{2}, n_{3}-j_{3}\right),
$$

where $a\left(j_{1}, j_{2}, j_{3}\right)$ 's are real constants such that

$$
\sum_{\mathbf{j}=-\infty}^{\infty}\left|a\left(j_{1}, j_{2}, j_{3}\right)\right|<\infty
$$

and $\left\{e\left(n_{1}, n_{2}, n_{3}\right) ; n_{1}, n_{2}, n_{3} \in Z\right\}$ is a sequence of $i . i . d$. real-valued random variables with mean zero and finite variance $\sigma^{2}$.

AsSUMPTION 2 Let $A^{0}$ and $B^{0}$ be arbitrary real numbers and $\left|A^{0}\right|+\left|B^{0}\right|>0$.

THEOREM 1 Under Assumptions 1 and 2, the LSEs of the parameters of model (1) are strongly consistent.

Proof of Theorem 1 See Appendix A.

Now, we obtain the asymptotic distribution of the LSEs of the parameters of model (1). First, we consider the case $p=1$, and then we generalize the result for arbitrary $p$. We use the following notation. The first derivative of $Q_{N}(\boldsymbol{\theta})$ is a $1 \times 5$ vector as

$$
Q_{N}^{\prime}(\boldsymbol{\theta})=\left[\frac{\partial Q_{N}(\boldsymbol{\theta})}{\partial A}, \frac{\partial Q_{N}(\boldsymbol{\theta})}{\partial B}, \frac{\partial Q_{N}(\boldsymbol{\theta})}{\partial \lambda_{1}}, \frac{\partial Q_{N}(\boldsymbol{\theta})}{\partial \lambda_{2}}, \frac{\partial Q_{N}(\boldsymbol{\theta})}{\partial \lambda_{3}}\right],
$$

and $Q_{N}^{\prime \prime}(\boldsymbol{\theta})$ is the $5 \times 5$ second derivative matrix of $Q_{N}(\boldsymbol{\theta})$. Therefore, expanding $Q_{N}^{\prime}(\hat{\boldsymbol{\theta}})$ around $\boldsymbol{\theta}^{0}$, we obtain

$$
Q_{N}^{\prime}(\hat{\boldsymbol{\theta}})-Q_{N}^{\prime}\left(\boldsymbol{\theta}^{0}\right)=\left(\hat{\boldsymbol{\theta}}-\boldsymbol{\theta}^{0}\right) Q_{N}^{\prime \prime}(\overline{\boldsymbol{\theta}}),
$$

where $\overline{\boldsymbol{\theta}}$ is a point on the line joining between $\hat{\boldsymbol{\theta}}$ and $\boldsymbol{\theta}^{0}$. As $Q_{N}^{\prime}(\hat{\boldsymbol{\theta}})=\mathbf{0}$, therefore equation (7) can be written as

$$
\left(\hat{\boldsymbol{\theta}}-\boldsymbol{\theta}^{0}\right) \mathbf{D}^{-1}=-Q_{N}^{\prime}\left(\boldsymbol{\theta}^{0}\right) \mathbf{D}\left[\mathbf{D} Q_{N}^{\prime \prime}(\overline{\boldsymbol{\theta}}) \mathbf{D}\right]^{-1}
$$

where

$$
\mathbf{D}=\left[\begin{array}{ccccc}
N^{-(1 / 2)} & 0 & 0 & 0 & 0 \\
0 & N^{-(1 / 2)} & 0 & 0 & 0 \\
0 & 0 & N_{1}^{-1} N^{-(1 / 2)} & 0 & 0 \\
0 & 0 & 0 & N_{2}^{-1} N^{-(1 / 2)} & 0 \\
0 & 0 & 0 & 0 & N_{3}^{-1} N^{-(1 / 2)}
\end{array}\right]
$$

We will show that $\mathbf{D} Q_{N}^{\prime \prime}(\overline{\boldsymbol{\theta}}) \mathbf{D}$ is a full rank matrix a.s. for large $N_{(1)}$, therefore for large $N_{(1)}$, $\mathbf{D} Q_{N}^{\prime \prime}(\overline{\boldsymbol{\theta}}) \mathbf{D}$ is invertible a.s. 
The different elements of $Q_{N}^{\prime}\left(\boldsymbol{\theta}^{0}\right) \mathbf{D}$ are as follows:

$$
\begin{aligned}
\frac{\partial}{\partial A} Q_{N}\left(\boldsymbol{\theta}^{0}\right) & =-2 \sum_{\mathbf{n}=\mathbf{1}}^{N} X(\mathbf{n}) \cos \left(\mathbf{n} \cdot \lambda^{0}\right), \\
\frac{\partial}{\partial B} Q_{N}\left(\boldsymbol{\theta}^{0}\right) & =-2 \sum_{\mathbf{n}=\mathbf{1}}^{N} X(\mathbf{n}) \sin \left(\mathbf{n} \cdot \lambda^{0}\right), \\
\frac{\partial}{\partial \lambda_{k}} Q_{N}\left(\boldsymbol{\theta}^{0}\right) & =2 \sum_{\mathbf{n}=\mathbf{1}}^{N} n_{k} X(\mathbf{n})\left[A^{0} \sin \left(\mathbf{n} \cdot \lambda^{0}\right)-B^{0} \cos \left(\mathbf{n} \cdot \lambda^{0}\right)\right], \text { for } k=1,2,3 .
\end{aligned}
$$

We need the following trigonometric results for $\lambda_{1}, \lambda_{2}, \lambda_{3} \in(0, \pi)$ :

$$
\begin{aligned}
& \lim _{N_{(1)} \rightarrow \infty} \frac{1}{N} \sum_{\mathbf{n}=\mathbf{1}}^{N} \cos ^{2}(\mathbf{n} \cdot \lambda)=\lim _{N_{(1)} \rightarrow \infty} \frac{1}{N} \sum_{\mathbf{n}=\mathbf{1}}^{N} \sin ^{2}(\mathbf{n} \cdot \lambda)=\frac{1}{2}, \\
& \lim _{N_{(1)} \rightarrow \infty} \frac{1}{N} \sum_{\mathbf{n}=\mathbf{1}}^{N} \cos (\mathbf{n} \cdot \lambda) \sin (\mathbf{n} \cdot \lambda)=0,
\end{aligned}
$$

and for $k=1,2,3$

$$
\begin{aligned}
& \lim _{N_{(1)} \rightarrow \infty} \frac{1}{N_{k} N} \sum_{\mathbf{n}=\mathbf{1}}^{N} n_{k} \cos ^{2}(\mathbf{n} \cdot \lambda)=\lim _{N_{(1)} \rightarrow \infty} \frac{1}{N_{k} N} \sum_{\mathbf{n}=\mathbf{1}}^{N} n_{k} \sin ^{2}(\mathbf{n} \cdot \lambda)=\frac{1}{4}, \\
& \lim _{N_{(1)} \rightarrow \infty} \frac{1}{N_{k}^{2} N} \sum_{\mathbf{n}=\mathbf{1}}^{N} n_{k}^{2} \cos ^{2}(\mathbf{n} \cdot \lambda)=\lim _{N_{(1)} \rightarrow \infty} \frac{1}{N_{k}^{2} N} \sum_{\mathbf{n}=\mathbf{1}}^{N} n_{k}^{2} \sin ^{2}(\mathbf{n} \cdot \lambda)=\frac{1}{6} .
\end{aligned}
$$

Results (9)-(12) can be found in Mangulis [24]. Let us look at the matrix: $\lim _{N_{(1)} \rightarrow \infty}\left[\mathbf{D} Q_{N}^{\prime \prime}\left(\boldsymbol{\theta}^{0}\right) \mathbf{D}\right]$. The $(1,1)$ th element of the limiting matrix can be written as:

$$
\lim _{N_{(1)} \rightarrow \infty} \frac{1}{N} \frac{\partial^{2} Q_{N}\left(\boldsymbol{\theta}^{\mathbf{0}}\right)}{\partial A^{2}}=\lim _{N_{(1)} \rightarrow \infty} \frac{2}{N} \sum_{\mathbf{n}=1}^{N} \cos ^{2}(\mathbf{n} \cdot \lambda)=1 \quad \text { (using (9)). }
$$

Similarly, using equations (9)-(12), it follows that

$$
\lim _{N_{(1)} \rightarrow \infty}\left[\mathbf{D} Q_{N}^{\prime \prime}\left(\boldsymbol{\theta}^{0}\right) \mathbf{D}\right]=\mathbf{\Sigma},
$$

where

$$
\boldsymbol{\Sigma}=\left[\begin{array}{ll}
\boldsymbol{\Sigma}_{11} & \boldsymbol{\Sigma}_{12} \\
\boldsymbol{\Sigma}_{21} & \boldsymbol{\Sigma}_{22}
\end{array}\right]
$$

and

$$
\begin{gathered}
\boldsymbol{\Sigma}_{11}=\mathbf{I}_{2 \times 2}, \quad \boldsymbol{\Sigma}_{12}=\boldsymbol{\Sigma}_{21}^{T}=\frac{1}{2}\left[\begin{array}{ccc}
B^{0} & B^{0} & B^{0} \\
-A^{0} & -A^{0} & -A^{0}
\end{array}\right] \\
\boldsymbol{\Sigma}_{22}=\left(A^{0^{2}}+B^{0^{2}}\right)\left[\begin{array}{ccc}
\frac{1}{3} & \frac{1}{4} & \frac{1}{4} \\
\frac{1}{4} & \frac{1}{3} & \frac{1}{4} \\
\frac{1}{4} & \frac{1}{4} & \frac{1}{3}
\end{array}\right] .
\end{gathered}
$$


As $\overline{\boldsymbol{\theta}}$ converges to $\boldsymbol{\theta}^{0},\left[\mathbf{D} Q_{N}^{\prime \prime}(\overline{\boldsymbol{\theta}}) \mathbf{D}\right]$ converges to $\left[\mathbf{D} Q_{N}^{\prime \prime}\left(\boldsymbol{\theta}^{0}\right) \mathbf{D}\right]=\boldsymbol{\Sigma}>\boldsymbol{0}$. So $\left[\mathbf{D} Q_{N}^{\prime \prime}(\overline{\boldsymbol{\theta}}) \mathbf{D}\right]$, is a full rank matrix a.s. for large $N_{(1)}$. Now, using the central limit theorem of the linear process [25, pp. 251-256] and using the earlier mentioned trigonometric results, it follows that $\left[Q_{N}^{\prime}\left(\boldsymbol{\theta}^{0}\right) \mathbf{D}\right]$ converges to a 5-variate normal distribution, with mean vector zero and dispersion matrix $2 \sigma^{2} c \boldsymbol{\Sigma}$, where

$$
c=\left|\sum_{\mathbf{j}=-\infty}^{\infty} a(\mathbf{j}) \mathrm{e}^{-i\left(\mathbf{j} \cdot \lambda^{0}\right)}\right|^{2},
$$

and $\boldsymbol{\Sigma}$ is defined earlier. Therefore, from equation (8), we have the following result.

THEOREM 2 Under Assumptions 1 and 2, the limiting distribution of $\left\{N^{(1 / 2)}\left(\hat{A}-A^{0}\right), N^{(1 / 2)}\left(\hat{B}-B^{0}\right), N_{1} N^{(1 / 2)}\left(\hat{\lambda}_{1}-\lambda_{1}^{0}\right), N_{2} N^{(1 / 2)}\left(\hat{\lambda}_{2}-\lambda_{2}^{0}\right), N_{3} N^{(1 / 2)}\left(\hat{\lambda}_{3}-\lambda_{3}^{0}\right)\right\}$

as $N_{(1)} \rightarrow \infty$ is a 5-variate normal distribution with mean vector zero and dispersion matrix $2 \sigma^{2} c \boldsymbol{\Sigma}^{-1}$, where $\boldsymbol{\Sigma}^{-1}$ has the following structure

$$
\Sigma^{-1}=\frac{1}{\left(A^{0^{2}}+B^{0^{2}}\right)}\left[\begin{array}{ccccc}
A^{0^{2}}+10 B^{0^{2}} & -9 A^{0} B^{0} & -6 B^{0} & -6 B^{0} & -6 B^{0} \\
-9 A^{0} B^{0} & 10 A^{0^{2}}+B^{0^{2}} & 6 A^{0} & 6 A^{0} & 6 A^{0} \\
-6 B^{0} & 6 A^{0} & 12 & 0 & 0 \\
-6 B^{0} & 6 A^{0} & 0 & 12 & 0 \\
-6 B^{0} & 6 A^{0} & 0 & 0 & 12
\end{array}\right] .
$$

For the general model (1), the following assumption is required.

Assumption 3 Let $A_{1}^{0}, \ldots, A_{p}^{0}$ and $B_{1}^{0}, \ldots, B_{p}^{0}$ are arbitrary real numbers such that $A_{j}^{0}$ and $B_{j}^{0}$ are not simultaneously equal to zero for all $j$. The frequencies $\lambda_{1 j}^{0}, \ldots, \lambda_{p j}^{0}, j=1,2,3$, are distinct.

Then the result for the general model (1) can be stated as follows.

THEOREM 3 Under Assumptions 1 and 3, the limiting distribution of

$$
\begin{aligned}
& \left\{N^{(1 / 2)}\left(\hat{A}_{k}-A_{k}^{0}\right), N^{(1 / 2)}\left(\hat{B}_{k}-B_{k}^{0}\right), N_{1} N^{(1 / 2)}\left(\hat{\lambda}_{k 1}-\lambda_{k 1}^{0}\right), N_{2} N^{(1 / 2)}\left(\hat{\lambda}_{k 2}-\lambda_{k 2}^{0}\right),\right. \\
& \left.\quad N_{3} N^{(1 / 2)}\left(\hat{\lambda}_{k 3}-\lambda_{k 3}^{0}\right)\right\}
\end{aligned}
$$

as $N_{(1)} \rightarrow \infty$ is a 5-variate normal distribution with mean vector zero and dispersion matrix $2 \sigma^{2} c_{k} \boldsymbol{\Sigma}_{k}^{-1}$, where the expressions for $c_{k}$ and $\boldsymbol{\Sigma}_{k}^{-1}$ can be obtained by replacing $A^{0}, B^{0}$, $\lambda_{1}^{0}, \lambda_{2}^{0}, \lambda_{3}^{0}$ with $A_{k}^{0}, B_{k}^{0}, \lambda_{k 1}^{0}, \lambda_{k 2}^{0}, \lambda_{k 3}^{0}$ respectively. Moreover, $\left(\hat{A}_{k}, \hat{B}_{k}, \hat{\lambda}_{k 1}, \hat{\lambda}_{k 2}, \hat{\lambda}_{k 3}\right)$ and $\left(\hat{A}_{m}, \hat{B}_{m}, \hat{\lambda}_{m 1}, \hat{\lambda}_{m 2}, \hat{\lambda}_{m 3}\right)$ are asymptotically independent for $k \neq m$.

\section{Consistency and asymptotic distribution of the ALSEs}

In this section, we consider the ALSEs of the different unknown parameters. Here also first we consider model (1) with $p=1$. As in section 3, we suppress $k$ for brevity. The ALSEs of 
$\lambda_{1}, \lambda_{2}$ and $\lambda_{3}$ can be obtained by maximizing the periodogram function

$$
I(\boldsymbol{\lambda})=\frac{1}{N}\left|\sum_{\mathbf{n}=\mathbf{1}}^{N} y(\mathbf{n}) \mathrm{e}^{-i(\mathbf{n} \cdot \lambda)}\right|^{2}
$$

If $\tilde{\lambda}=\left(\tilde{\lambda}_{1}, \tilde{\lambda}_{2}, \tilde{\lambda}_{3}\right)$ maximizes $I(\lambda)$, then $\tilde{\lambda}$ is called the ALSE of $\lambda^{0}$. Once we obtain $\tilde{\lambda}$, along the same lines as Hannan [6] and Walker [8], we propose the ALSEs of $A$ and $B$ as

$$
\begin{aligned}
& \tilde{A}=\frac{2}{N} \sum_{\mathbf{n}=\mathbf{1}}^{N} y(\mathbf{n}) \cos (\tilde{\boldsymbol{\lambda}} \cdot \mathbf{n}), \\
& \tilde{B}=\frac{2}{N} \sum_{\mathbf{n}=\mathbf{1}}^{N} y(\mathbf{n}) \sin (\tilde{\lambda} \cdot \mathbf{n}) .
\end{aligned}
$$

We have the following results.

THEOREM 4 Under Assumptions 1 and 2, the ALSE, $\tilde{\lambda}$, converges to $\lambda^{0}$ almost surely.

Proof of Theorem 4 See Appendix B.

THEOREM 5 Under Assumptions 1 and 2, the ALSEs, $\tilde{A}$ and $\tilde{B}$, as defined in equations (15) and (16), are strongly consistent estimators of $A^{0}$ and $B^{0}$, respectively.

Proof of Theorem 5 See Appendix B.

THEOREM 6 Under Assumptions 1 and 2, the limiting distribution of

$$
\begin{aligned}
& \left\{N^{(1 / 2)}\left(\tilde{A}-A^{0}\right), N^{(1 / 2)}\left(\tilde{B}-B^{0}\right), N_{1} N^{(1 / 2)}\left(\tilde{\lambda}_{1}-\lambda_{1}^{0}\right), N_{2} N^{(1 / 2)}\left(\tilde{\lambda}_{2}-\lambda_{2}^{0}\right),\right. \\
& \left.\quad N_{3} N^{(1 / 2)}\left(\tilde{\lambda}_{3}-\lambda^{0}\right)\right\}
\end{aligned}
$$

as $N_{(1)} \rightarrow \infty$, is same as that of the LSEs.

\section{Multidimensional model}

In this section, we consider the m-D frequency model. For avoiding repetitions, we mainly consider the LSEs; similar results follow for the ALSEs also. For brevity, we use the following 
notation in this section, and they should not create any confusion with the previous sections.

$$
\begin{array}{cl}
\mathbf{n}=\left(n_{1}, \ldots, n_{m}\right) & \mathbf{j}=\left(j_{1}, \ldots, j_{m}\right) \\
\mathbf{n}-\mathbf{j}=\left(n_{1}-j_{1}, \ldots, n_{m}-j_{m}\right) & \lambda=\left(\lambda_{1}, \ldots, \lambda_{m}\right) \\
\lambda_{k}=\left(\lambda_{k 1}, \ldots, \lambda_{k m}\right) & \lambda_{k}^{0}=\left(\lambda_{k 1}^{0}, \ldots, \lambda_{k m}^{0}\right) \\
\boldsymbol{\theta}_{k}=\left(A_{k}, B_{k}, \lambda_{k 1}, \ldots, \lambda_{k m}\right) & \boldsymbol{\theta}_{k}^{0}=\left(A_{k}^{0}, B_{k}^{0}, \lambda_{k 1}^{0}, \ldots, \lambda_{k m}^{0}\right), \quad \text { for } k=1, \ldots, p \\
\boldsymbol{\theta}=\left(\boldsymbol{\theta}_{1}, \ldots, \boldsymbol{\theta}_{p}\right) & \boldsymbol{\theta}^{0}=\left(\boldsymbol{\theta}_{1}^{0}, \ldots, \boldsymbol{\theta}_{p}^{0}\right) \\
N=N_{1}, \ldots, N_{m} & N_{(1)}=\min \left\{N_{1}, \ldots, N_{m}\right\} \\
\sum_{\mathbf{n}=\mathbf{1}}^{N}=\sum_{n_{1}=1}^{N_{1}} \ldots \sum_{n_{m}=1}^{N_{m}} & \sum_{\mathbf{j}=-\infty}^{\infty}=\sum_{j_{1}=-\infty}^{\infty} \ldots \sum_{j_{m}=-\infty}^{\infty} \\
\sup _{\lambda}=\sup _{\lambda_{1}, \ldots, \lambda_{m}} & \\
\mu(\boldsymbol{\theta})=\sum_{k=1}^{p}\left(A_{k} \cos \left(n_{1} \lambda_{k 1}+\ldots+n_{m} \lambda_{k m}\right)+B_{k} \sin \left(n_{1} \lambda_{k 1}+\ldots+n_{m} \lambda_{k m}\right)\right) \\
=\sum_{k=1}^{p}\left(A_{k} \cos \left(\mathbf{n} \cdot \lambda_{k}\right)+B_{k} \sin \left(\mathbf{n} \cdot \lambda_{k}\right)\right) .
\end{array}
$$

With the above mentioned notation, the most general m-D sinusoidal model is as follows:

$$
y(\mathbf{n})=\sum_{k=1}^{p}\left[A_{k}^{0} \cos \left(\mathbf{n} \cdot \lambda_{k}^{0}\right)+B_{k}^{0} \sin \left(\mathbf{n} \cdot \lambda_{k}^{0}\right)\right]+X(\mathbf{n}) .
$$

Here, $A_{k}^{0}, B_{k}^{0}$ and $\lambda_{k}^{0}$ satisfy similar assumptions as defined in section 1 and $X(\mathbf{n})$ satisfies assumption 4 , which is an obvious modification of Assumption 1.

ASSUMPTION $4 \quad X(\mathbf{n})$ can be represented as follows for $n_{1}, \ldots, n_{m} \in Z$ :

$$
X(\mathbf{n})=\sum_{\mathbf{j}=-\infty}^{\infty} a(\mathbf{j}) \mathrm{e}(\mathbf{n}-\mathbf{j})
$$

where $a(\mathbf{j})$ 's are real constants such that

$$
\sum_{\mathbf{j}=-\infty}^{\infty}|a(\mathbf{j})|<\infty
$$

and $\left\{e(\mathbf{n}) ; n_{1}, \ldots, n_{m} \in Z\right\}$ is a $m-D$ sequence of i.i.d. random variables with mean zero and finite variance $\sigma^{2}$. 
First, we consider LSEs of the unknown parameters and they can be obtained by minimizing

$$
Q_{N}(\boldsymbol{\theta})=\sum_{\mathbf{n}=\mathbf{1}}^{N}(y(\mathbf{n})-\mu(\boldsymbol{\theta}))^{2},
$$

with respect to the unknown parameters. For notational simplicity, let us consider $p=1$, i.e. we consider the following model

$$
y(\mathbf{n})=A^{0} \cos \left(\mathbf{n} \cdot \lambda^{0}\right)+B^{0} \sin \left(\mathbf{n} \cdot \lambda^{0}\right)+X(\mathbf{n}) .
$$

If $\hat{A}, \hat{B}, \hat{\lambda}\left(=\left(\hat{\lambda}_{1}, \ldots, \hat{\lambda}_{m}\right)\right)$ denote the LSEs of $A^{0}, B^{0}$ and $\lambda^{0}$, respectively, then we have the following results (the proofs are available in Appendix C).

THEOREM 7 Under Assumptions 2 and 4, $\hat{A}, \hat{B}$ and $\hat{\lambda}$ are strongly consistent estimators of $A^{0}, B^{0}$ and $\lambda^{0}$, respectively.

THEOREM 8 Under Assumptions 2 and 4, the limiting distribution of

$$
\left\{N^{(1 / 2)}\left(\hat{A}-A^{0}\right), N^{(1 / 2)}\left(\hat{B}-B^{0}\right), N_{1} N^{(1 / 2)}\left(\hat{\lambda}_{1}-\lambda_{1}^{0}\right), \ldots, N_{m} N^{(1 / 2)}\left(\hat{\lambda}_{m}-\lambda_{m}^{0}\right)\right\}
$$

as $N_{(1)} \rightarrow \infty$ is a $(m+2)$-variate normal distribution with mean vector zero and dispersion matrix $2 \sigma^{2} c \boldsymbol{\Sigma}^{-1}$, where the matrix $\boldsymbol{\Sigma}^{-1}$ has the following structure

$$
\Sigma^{-1}=\frac{1}{\left(A^{0^{2}}+B^{0^{2}}\right)}\left[\begin{array}{ccccc}
A^{0^{2}}+(3 m+1) B^{0^{2}} & -3 m A^{0} B^{0} & -6 B^{0} & \ldots & -6 B^{0} \\
-3 m A^{0} B^{0} & (3 m+1) A^{0^{2}}+B^{0^{2}} & 6 A^{0} & \ldots & 6 A^{0} \\
-6 B^{0} & 6 A^{0} & 12 & \ldots & 0 \\
\vdots & \vdots & \vdots & \ddots & \vdots \\
-6 B^{0} & 6 A^{0} & 0 & \ldots & 12
\end{array}\right],
$$

and $c$ is same as defined in (13), but with the new notation.

This distribution says that provided all $N_{k}$ 's are same, all frequencies can be estimated with equal accuracy whatever be the dimension of the model, whereas the asymptotic variances of $A$ and $B$ increase with increasing number of dimension. In addition, the asymptotic dependence between $A$ and every frequency is same in each dimension. The same is true for $B$ also. Moreover, Theorem 8 can be extended for arbitrary $p$ in the same way as Theorem 3 .

\section{Conclusions}

In this article, we first consider the 3-D and then the m-D sinusoidal models in the presence of additive stationary errors. We have considered two different estimators mainly the LSEs and the estimators obtained by maximizing the periodogram function, known as the ALSEs. We have obtained the consistency and the asymptotic distributions of both the estimators, and it is observed that the two estimators are equivalent. Our results generalize several 1-D or 2-D results to the m-D case. One important problem that we did not address is the computational aspects of the two estimators. It is well known that even for one or two dimensions, the computational problem is quite challenging; therefore, it is expected that for higher dimension, the problem will be more challenging. More work is needed in that direction. 


\section{Acknowledgements}

The authors would like to thank the referees for their valuable comments which certainly helped to improve the earlier draft of the manuscript and also the editor-in-chief Professor Dr. Olaf Bunke for his encouragement.

\section{Appendix A}

We need the following lemmas to prove the necessary results.

\section{LEMMA 1 Let us denote}

$$
S_{\delta, M}=\left\{\boldsymbol{\theta} ; \boldsymbol{\theta}=\left(A, B, \lambda_{1}, \lambda_{2}, \lambda_{3}\right),\left|\boldsymbol{\theta}-\boldsymbol{\theta}^{0}\right| \geq 5 \delta,\left|A-A^{0}\right| \leq M,\left|B-B^{0}\right| \leq M\right\},
$$

for any $\delta>0$ and for some $M<\infty$, if

$$
\liminf _{N_{(1)} \rightarrow \infty} \inf _{\boldsymbol{\theta} \in S_{\delta, M}} \frac{1}{N}\left[Q_{N}(\boldsymbol{\theta})-Q_{N}\left(\boldsymbol{\theta}^{0}\right)\right]>0, \quad \text { a.s. }
$$

then $\hat{\boldsymbol{\theta}}$ is a strongly consistent estimator of $\boldsymbol{\theta}^{0}$.

Proof of Lemma 1 The proof is similar to that of Lemma 1 of Kundu and Nandi [21], see also $\mathrm{Wu}[26]$.

Lemma 2 If $X(\mathbf{n})$ satisfy Assumption 1, then as $N_{(1)} \rightarrow \infty$,

$$
\sup _{\lambda}\left|\frac{1}{N} \sum_{\mathbf{n}=\mathbf{1}}^{N} X(\mathbf{n}) \mathrm{e}^{i(\mathbf{n} \cdot \lambda)}\right| \rightarrow 0 \quad \text { a.s. }
$$

Proof of Lemma 2 Observe that

$$
\begin{aligned}
\sup _{\lambda} \frac{1}{N}\left|\sum_{\mathbf{n}=\mathbf{1}}^{N} X(\mathbf{n}) \mathrm{e}^{i(\mathbf{n} \cdot \lambda)}\right| & =\sup _{\lambda} \frac{1}{N}\left|\sum_{\mathbf{n}=\mathbf{1}}^{N} \sum_{\mathbf{j}=-\infty}^{\infty} a(\mathbf{j}) \mathrm{e}(\mathbf{n}-\mathbf{j}) \mathrm{e}^{i(\mathbf{n} \cdot \lambda)}\right| \\
& \leq \frac{1}{N} \sum_{\mathbf{j}=-\infty}^{\infty}|a(\mathbf{j})| \sup _{\lambda}\left|\sum_{\mathbf{n}=\mathbf{1}}^{N} \mathrm{e}(\mathbf{n}-\mathbf{j}) \mathrm{e}^{i(\mathbf{n} \cdot \lambda)}\right| .
\end{aligned}
$$

Therefore,

$$
\begin{aligned}
E\left(\sup _{\lambda} \frac{1}{N}\left|\sum_{\mathbf{n}=\mathbf{1}}^{N} X(\mathbf{n}) \mathrm{e}^{i(\mathbf{n} \cdot \boldsymbol{\lambda})}\right|\right) & \leq \frac{1}{N} \sum_{\mathbf{j}=-\infty}^{\infty}|a(\mathbf{j})| E\left(\sup _{\lambda}\left|\sum_{\mathbf{n}=\mathbf{1}}^{N} \mathrm{e}(\mathbf{n}-\mathbf{j}) \mathrm{e}^{i(\mathbf{n} \cdot \lambda)}\right|\right) \\
& \leq \sum_{\mathbf{j}=-\infty}^{\infty}|a(\mathbf{j})| \frac{1}{N}\left\{E\left(\sup _{\lambda}\left|\sum_{\mathbf{n}=\mathbf{1}}^{N} \mathrm{e}(\mathbf{n}-\mathbf{j}) \mathrm{e}^{i(\mathbf{n} \cdot \boldsymbol{\lambda})}\right|\right)^{2}\right\}^{1 / 2} .
\end{aligned}
$$


Now consider

$$
\begin{aligned}
& \frac{1}{N}\left\{E\left(\sup _{\lambda}\left|\sum_{\mathbf{n}=1}^{N} \mathrm{e}(\mathbf{n}-\mathbf{j}) \mathrm{e}^{i(\mathbf{n} \cdot \lambda)}\right|^{2}\right)\right\}^{1 / 2}
\end{aligned}
$$

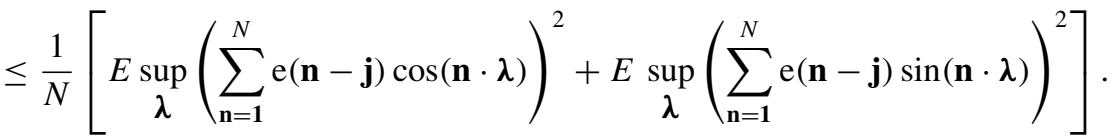

Using the 1-D result of Kundu [4, p. 225], it follows that the right-hand side of equation (22) is of the order

$$
\frac{1}{N}\left[N+N^{(3 / 2)}\right]^{(1 / 2)}=N^{-(1 / 4)}
$$

Therefore, the right-hand side of equation (21) is $O\left(N^{-1 / 4}\right)$. Now if we take any subsequence $\left\{N^{\delta}\right\}=\left\{N_{1}^{\delta} \cdot N_{2}^{\delta} \cdot N_{3}^{\delta}\right\}$ of $\{N\}$, where $\delta>4$, then the right-hand side of equation (21) is summable over that subsequence. Let us take $\delta=5$, therefore by Chebyshev's inequality and using the Borel-Cantelli lemma, we can say that

$$
\sup _{\lambda} \frac{1}{N^{5}}\left|\sum_{\mathbf{n}=1}^{N^{5}} X(\mathbf{n}) \mathrm{e}^{i(\mathbf{n} \cdot \lambda)}\right| \longrightarrow 0 \quad \text { a.s. }
$$

as $N_{(1)} \rightarrow \infty$, here $\sum_{\mathbf{n}=1}^{N^{5}}=\sum_{n_{1}=1}^{N_{1}^{5}} \sum_{n_{2}=1}^{N_{2}^{5}} \sum_{n_{3}=1}^{N_{3}^{5}}$. Now consider

$$
\frac{1}{N^{5}} \sup _{\lambda} \sup _{R}\left|\sum_{n_{1}=1}^{R_{1}} \sum_{n_{2}=1}^{R_{2}} \sum_{n_{3}=1}^{R_{3}} X(\mathbf{n}) \mathrm{e}^{i(\mathbf{n} \cdot \lambda)}-\sum_{n_{1}=1}^{N_{1}^{5}} \sum_{n_{2}=1}^{N_{2}^{5}} \sum_{n_{3}=1}^{N_{3}^{5}} X(\mathbf{n}) \mathrm{e}^{i(\mathbf{n} \cdot \lambda)}\right|
$$

where $R$ denotes the range of $R_{1}, R_{2}$ and $R_{3}$ and $N_{1}^{5}<R_{1} \leq\left(N_{1}+1\right)^{5}, N_{2}^{5}<R_{2} \leq\left(N_{2}+1\right)^{5}$, $N_{3}^{5}<R_{3} \leq\left(N_{3}+1\right)^{5}$. Observe that

$$
\begin{aligned}
& \frac{1}{N^{5}} \sup _{\lambda} \sup _{R}\left|\sum_{n_{1}=1}^{R_{1}} \sum_{n_{2}=1}^{R_{2}} \sum_{n_{3}=1}^{R_{3}} X(\mathbf{n}) \mathrm{e}^{i(\mathbf{n} \cdot \lambda)}-\sum_{n_{1}=1}^{N_{1}^{5}} \sum_{n_{2}=1}^{N_{2}^{5}} \sum_{n_{3}=1}^{N_{3}^{5}} X(\mathbf{n}) \mathrm{e}^{i(\mathbf{n} \cdot \lambda)}\right| \\
& \leq \frac{1}{N^{5}} \sup _{\lambda} \sup _{R_{3}}\left|\sum_{n_{1}=1}^{N_{1}^{5}} \sum_{n_{2}=1}^{N_{2}^{5}} \sum_{n_{3}=N_{3}^{5}+1}^{R_{3}} X(\mathbf{n}) \mathrm{e}^{i(\mathbf{n} \cdot \lambda)}\right|+\text { (two similar terms) } \\
& +\frac{1}{N^{5}} \sup _{\lambda} \sup _{R_{2}, R_{3}}\left|\sum_{n_{1}=1}^{N_{1}^{5}} \sum_{n_{2}=N_{2}^{5}+1}^{R_{2}} \sum_{n_{3}=N_{3}^{5}+1}^{R_{3}} X(\mathbf{n}) \mathrm{e}^{i(\mathbf{n} \cdot \lambda)}\right|+\text { (two similar terms) } \\
& +\frac{1}{N^{5}} \sup _{\lambda}\left|\sum_{n_{1}=N_{1}^{5}+1}^{\left(N_{1}+1\right)^{5}} \sum_{n_{2}=N_{2}^{5}+1}^{\left(N_{2}+1\right)^{5}} \sum_{n_{3}=N_{3}^{5}+1}^{N_{3}+1}{ }^{5} X(\mathbf{n}) \mathrm{e}^{i(\mathbf{n} \cdot \lambda)}\right|
\end{aligned}
$$


Now consider

$$
\begin{aligned}
& \frac{1}{N^{5}} \sup _{\lambda} \sup _{R_{3}}\left|\sum_{n_{1}=1}^{N_{1}^{5}} \sum_{n_{2}=1}^{N_{2}^{5}} \sum_{n_{3}=N_{3}^{5}+1}^{R_{3}} X(\mathbf{n}) \mathrm{e}^{i(\mathbf{n} \cdot \lambda)}\right| \\
& \quad \leq \frac{1}{N_{3}^{5}} \sup _{N_{3}<R_{3} \leq\left(N_{3}+1\right)^{5}} \sum_{n_{3}=N_{3}^{5}+1}^{R_{3}}\left|Y\left(n_{3}\right)\right| \leq \frac{1}{N_{3}^{5}} \sum_{n_{3}=N_{3}^{5}+1}^{\left(N_{3}+1\right)^{5}}\left|Y\left(n_{3}\right)\right|,
\end{aligned}
$$

where

$$
\left|Y\left(n_{3}\right)\right|=\sup _{\lambda_{1}, \lambda_{2}}\left|\frac{1}{\left(N_{1} N_{2}\right)^{5}} \sum_{n_{1}=1}^{N_{1}^{5}} \sum_{n_{2}=1}^{N_{2}^{5}} X(\mathbf{n}) \mathrm{e}^{i\left(n_{1} \lambda_{1}+n_{2} \lambda_{2}\right)}\right| .
$$

From Kundu and Nandi [21], it follows that $E\left|Y\left(n_{3}\right)\right|=O\left(N_{1} N_{2}\right)^{-(5 / 4)}$, therefore

$$
E\left(\frac{1}{N_{3}^{5}} \sum_{n_{3}=N_{3}^{5}+1}^{\left(N_{3}+1\right)^{5}}\left|Y\left(n_{3}\right)\right|\right) \leq O\left(\left(N_{1} N_{2}\right)^{-(5 / 4)} N_{3}^{-2}\right)
$$

Using the Borel-Cantelli lemma, it implies that

$$
\frac{1}{N^{5}} \sup _{\lambda} \sup _{R_{3}}\left|\sum_{n_{1}=1}^{N_{1}^{5}} \sum_{n_{2}=1}^{N_{2}^{5}} \sum_{n_{3}=N_{3}^{5}+1}^{R_{3}} X(\mathbf{n}) \mathrm{e}^{i(\mathbf{n} \cdot \lambda)}\right| \longrightarrow 0 \quad \text { a.s. }
$$

Now consider the term

$$
\frac{1}{N^{5}} \sup _{\lambda} \sup _{R_{2}, R_{3}}\left|\sum_{n_{1}=1}^{N_{1}^{5}} \sum_{n_{2}=N_{2}^{5}+1}^{R_{2}} \sum_{n_{3}=N_{3}^{5}+1}^{R_{3}} X(\mathbf{n}) \mathrm{e}^{i(\mathbf{n} \cdot \boldsymbol{\lambda})}\right| \leq \frac{1}{N_{2}^{5}} \sum_{n_{2}=N_{2}^{5}+1}^{\left(N_{2}+1\right)^{5}}\left|Z\left(n_{2}\right)\right|,
$$

where

$$
\left|Z\left(n_{2}\right)\right|=\frac{1}{N_{1}^{5} N_{3}^{5}} \sup _{\lambda_{1}, \lambda_{3}} \sup _{N_{3}<R_{3} \leq\left(N_{3}+1\right)^{5}}\left|\sum_{n_{1}=1}^{N_{1}^{5}} \sum_{n_{3}=N_{3}^{5}+1}^{R_{3}} X(\mathbf{n}) \mathrm{e}^{i\left(n_{1} \lambda_{1}+n_{3} \lambda_{3}\right)}\right| .
$$

It is observed in Kundu and Nandi [21] that

$$
E\left(\left|Z\left(n_{2}\right)\right|\right) \leq O\left(N_{1}^{-(5 / 4)} N_{3}^{-2}\right),
$$

therefore

$$
E\left(\frac{1}{N_{2}^{5}} \sum_{n_{2}=N_{2}^{5}+1}^{\left(N_{2}+1\right)^{5}}\left|Z\left(n_{2}\right)\right|\right) \leq O\left(N_{1}^{-(5 / 4)}\left(N_{2} N_{3}\right)^{-2}\right) .
$$

Again using the Borel-Cantelli lemma, it implies that

$$
\frac{1}{N^{5}} \sup _{\lambda} \sup _{R_{2}, R_{3}}\left|\sum_{n_{1}=1}^{N_{1}^{5}} \sum_{n_{2}=N_{2}^{5}+1}^{R_{2}} \sum_{n_{3}=N_{3}^{5}+1}^{R_{3}} X(\mathbf{n}) \mathrm{e}^{i(\mathbf{n} \cdot \boldsymbol{\lambda})}\right| \longrightarrow 0 \text { a.s. }
$$


Then, the term

$$
\frac{1}{N^{5}} \sup _{\lambda}\left|\sum_{n_{1}=N_{1}^{5}+1}^{\left(N_{1}+1\right)^{5}} \sum_{n_{2}=N_{2}^{5}+1}^{\left(N_{2}+1\right)^{5}} \sum_{n_{3}=N_{3}^{5}+1}^{\left(N_{3}+1\right)^{5}} X(\mathbf{n}) \mathrm{e}^{i(\mathbf{n} \cdot \lambda)}\right| \leq \frac{1}{N^{5}} \sum_{n_{1}=N_{1}^{5}+1}^{\left(N_{1}+1\right)^{5}} \sum_{n_{2}=N_{2}^{5}+1}^{\left(N_{2}+1\right)^{5}} \sum_{n_{3}=N_{3}^{5}+1}^{\left(N_{3}+1\right)^{5}}|X(\mathbf{n})| .
$$

As the mean-squared errors of the right-hand side of equation (23) is of the order $\mathrm{O}\left(\left(N_{1} N_{2} N_{3}\right)^{-2}\right)$, it also converges to zero using the Borel-Cantelli lemma and that proves the lemma.

Following the proof of Lemma 2, it can be shown that as $N_{(1)} \rightarrow \infty$,

$$
\sup _{\lambda}\left|\frac{1}{N_{1}^{k_{1}+1} N_{2}^{k_{2}+1} N_{3}^{k_{3}+1}} \sum_{\mathbf{n}=\mathbf{1}}^{N} n_{1}^{k_{1}} n_{2}^{k_{2}} n_{3}^{k_{3}} X(\mathbf{n}) \mathrm{e}^{i(\mathbf{n} \cdot \lambda)}\right| \longrightarrow 0 \quad \text { a.s. }
$$

for $k_{j}=1,2, \ldots$ and $j=1,2,3$.

Note that Lemma 2 is a very strong result. It extends several 1-D and 2-D results to the 3-D case.

Proof of Theorem 1 Note that

$$
\frac{1}{N}\left(Q_{N}(\boldsymbol{\theta})-Q_{N}\left(\boldsymbol{\theta}^{0}\right)\right)=\frac{1}{N} \sum_{\mathbf{n}=\mathbf{1}}^{N}\left(\mu\left(\boldsymbol{\theta}^{0}\right)-\mu(\boldsymbol{\theta})\right)^{2}+\frac{2}{N} \sum_{\mathbf{n}=\mathbf{1}}^{N} X(\mathbf{n})\left(\mu\left(\boldsymbol{\theta}^{0}\right)-\mu(\boldsymbol{\theta})\right) .
$$

Observe that the second term of the right-hand side of equation (25) converges to zero because of Lemma 2. Therefore, using Lemma 1, to prove the consistency of $\hat{\boldsymbol{\theta}}$, it remains to prove that

$$
\liminf _{N_{(1)} \rightarrow \infty} \inf _{\boldsymbol{\theta} \in S_{\delta, M}} f(\boldsymbol{\theta})>0 \quad \text { a.s., }
$$

where $f(\boldsymbol{\theta})=\frac{1}{N} \sum_{\mathbf{n}=\mathbf{1}}^{N}\left(\mu\left(\boldsymbol{\theta}^{0}\right)-\mu(\boldsymbol{\theta})\right)^{2}$. Consider the following sets

$$
\begin{aligned}
A_{\delta} & =\left\{\boldsymbol{\theta}: \boldsymbol{\theta}=\left(A, B, \lambda_{1}, \lambda_{2}, \lambda_{3}\right),\left|A-A^{0}\right| \geq \delta,|A| \leq M,|B| \leq M\right\}, \\
B_{\delta} & =\left\{\boldsymbol{\theta}: \boldsymbol{\theta}=\left(A, B, \lambda_{1}, \lambda_{2}, \lambda_{3}\right),\left|B-B^{0}\right| \geq \delta,|A| \leq M,|B| \leq M\right\}, \\
\Lambda_{k \delta} & =\left\{\boldsymbol{\theta}: \boldsymbol{\theta}=\left(A, B, \lambda_{1}, \lambda_{2}, \lambda_{3}\right),\left|\lambda_{k}-\lambda_{k}^{0}\right| \geq \delta,|A| \leq M,|B| \leq M\right\}, \quad k=1,2,3
\end{aligned}
$$

then $S_{\delta, M} \subset A_{\delta} \cup B_{\delta} \cup \Lambda_{1 \delta} \cup \Lambda_{2 \delta} \cup \Lambda_{3 \delta}$. Thus, to prove equation (26), it is enough to prove that

$$
\liminf _{N_{(1)} \rightarrow \infty} \inf _{\boldsymbol{\theta} \in R} f(\boldsymbol{\theta})>0 \quad \text { a.s., }
$$

where $R$ is any one of the sets defined in equation (27). First suppose $R=A_{\delta}$, then

$$
\liminf _{N_{(1)} \rightarrow \infty} \inf _{\boldsymbol{\theta} \in A_{\delta}} \frac{1}{N} \sum_{\mathbf{n}=\mathbf{1}}^{N}\left(\mu\left(\boldsymbol{\theta}^{0}\right)-\mu(\boldsymbol{\theta})\right)^{2}=\left(A^{0}-A\right)^{2} \lim _{N_{(1)} \rightarrow \infty} \sum_{\mathbf{n}=\mathbf{1}}^{N} \cos ^{2}(\mathbf{n} \cdot \lambda) \geq \frac{\delta^{2}}{2}>0 \quad \text { a.s. }
$$

Similarly, it can be proved for other sets. This proves the theorem. 


\section{Appendix B}

To prove Theorem 4, we need the following lemma.

LEMMA 3 Let us denote

$$
L_{c}=\left\{\lambda=\left(\lambda_{1}, \lambda_{2}, \lambda_{3}\right) ; \lambda_{1}, \lambda_{2}, \lambda_{3} \in(0, \pi),\left|\lambda-\lambda^{0}\right|>3 c\right\},
$$

for any $c>0$. If there exists $c>0$, such that

$$
\limsup _{N_{(1)} \rightarrow \infty} \sup _{\lambda \in L_{c}} \frac{1}{N}\left[I(\lambda)-I\left(\lambda^{0}\right)\right]<0 \quad \text { a.s. }
$$

then $\tilde{\lambda} \rightarrow \lambda^{0}$ a.s. as $N_{(1)} \rightarrow \infty$.

Proof of Lemma 3 The proof follows along the same line as Lemma 1 and it is omitted.

Proof of Theorem 4 Consider

$$
\begin{aligned}
\frac{1}{N}\left[I(\boldsymbol{\lambda})-I\left(\lambda^{0}\right)\right]= & \left.\frac{1}{N} \sum_{\mathbf{n}=\mathbf{1}}^{N}\left(A^{0} \cos \left(\mathbf{n} \cdot \lambda^{0}\right)+B^{0} \sin \left(\mathbf{n} \cdot \lambda^{0}\right)+X(\mathbf{n})\right) \cos (\mathbf{n} \cdot \boldsymbol{\lambda})\right]^{2} \\
& +\left[\frac{1}{N} \sum_{\mathbf{n}=\mathbf{1}}^{N}\left(A^{0} \cos \left(\mathbf{n} \cdot \lambda^{0}\right)+B^{0} \sin \left(\mathbf{n} \cdot \lambda^{0}\right)+X(\mathbf{n})\right) \sin (\mathbf{n} \cdot \boldsymbol{\lambda})\right]^{2} \\
& -\left[\frac{1}{N} \sum_{\mathbf{n}=1}^{N}\left(A^{0} \cos \left(\mathbf{n} \cdot \lambda^{0}\right)+B^{0} \sin \left(\mathbf{n} \cdot \lambda^{0}\right)+X(\mathbf{n})\right) \cos \left(\mathbf{n} \cdot \lambda^{0}\right)\right]^{2} \\
& -\left[\frac{1}{N} \sum_{\mathbf{n}=\mathbf{1}}^{N}\left(A^{0} \cos \left(\mathbf{n} \cdot \lambda^{0}\right)+B^{0} \sin \left(\mathbf{n} \cdot \lambda^{0}\right)+X(\mathbf{n})\right) \sin \left(\mathbf{n} \cdot \lambda^{0}\right)\right]^{2} .
\end{aligned}
$$

Let us write $L_{c}=L_{1 c} \cup L_{2 c} \cup L_{3 c}$, where

$$
L_{l c}=\left\{\lambda=\left(\lambda_{1}, \lambda_{2}, \lambda_{3}\right) ; \lambda_{1}, \lambda_{2}, \lambda_{3} \in(0, \pi),\left|\lambda_{k}-\lambda_{k}^{0}\right|>c\right\}, \quad l=1,2,3 .
$$

Now using Lemma 2 and the trigonometric identities, we have for $L_{l c}, l=1,2,3$,

$$
\begin{aligned}
& \limsup _{N_{(1)} \rightarrow \infty} \sup _{\lambda \in L_{l c}} \frac{1}{N}\left[I(\lambda)-I\left(\lambda^{0}\right)\right] \\
& \quad=-\limsup _{N_{(1)} \rightarrow \infty}\left\{\left[\frac{1}{N} \sum_{\mathbf{n}=\mathbf{1}}^{N} A^{0} \cos ^{2}\left(\mathbf{n} \cdot \lambda^{0}\right)\right]^{2}+\left[\frac{1}{N} \sum_{\mathbf{n}=\mathbf{1}}^{N} B^{0} \sin ^{2}\left(\mathbf{n} \cdot \lambda^{0}\right)\right]^{2}\right\} \\
& =-\frac{1}{4}\left(A^{0^{2}}+B^{0^{2}}\right)<0 \quad \text { a.s. }
\end{aligned}
$$

Therefore, the result follows using Lemma 3.

Lemma 5 Suppose $\tilde{\lambda}$ is the ALSE of $\lambda^{0}$ and $\mathbf{D}_{1}=\operatorname{diag}\left\{N_{1}^{-1}, N_{2}^{-1}, N_{3}^{-1}\right\}$, then

$$
\left(\tilde{\boldsymbol{\lambda}}-\lambda^{0}\right) \mathbf{D}_{1}^{-1} \longrightarrow \mathbf{0} \text { a.s. }
$$


Proof of Lemma 5 Let us denote the $1 \times 3$ first derivative vector of $\mathbf{I}(\lambda)$ by $\mathbf{I}^{\prime}(\lambda)$ and the $3 \times 3$ second derivative matrix by $\mathbf{I}^{\prime \prime}(\lambda)$. The multivariate Taylor series expansion of $\mathbf{I}^{\prime}(\lambda)$ around $\lambda^{0}$ yields

$$
\mathbf{I}^{\prime}(\tilde{\lambda})-\mathbf{I}^{\prime}\left(\lambda^{0}\right)=\left(\tilde{\lambda}-\lambda^{0}\right) \mathbf{I}^{\prime \prime}(\bar{\lambda}),
$$

where $\bar{\lambda}$ is a point between $\tilde{\lambda}$ and $\lambda^{0}$. As $\mathbf{I}^{\prime}(\tilde{\lambda})=\mathbf{0}$, we have

$$
\left(\tilde{\lambda}-\lambda^{0}\right) \mathbf{D}_{1}^{-1}=-\left[\frac{1}{N} \mathbf{I}^{\prime}\left(\lambda^{0}\right) \mathbf{D}_{1}\right]\left[\frac{1}{N} \mathbf{D}_{1} \mathbf{I}^{\prime \prime}(\bar{\lambda}) \mathbf{D}_{1}\right]^{-1} .
$$

Let us consider the different elements of $(1 / N) \mathbf{I}^{\prime}\left(\lambda^{0}\right) \mathbf{D}_{1}$. Note that the $k$ th element is

$$
\begin{aligned}
\frac{1}{N_{k} \cdot N} \frac{\partial \mathbf{I}\left(\lambda^{0}\right)}{\partial \lambda_{k}}= & \frac{2}{N_{k} \cdot N^{2}}\left[\sum_{\mathbf{n}=\mathbf{1}}^{N} y(\mathbf{n}) \cos \left(\mathbf{n} \cdot \lambda^{0}\right)\right] \cdot\left[-\sum_{\mathbf{n}=\mathbf{1}}^{N} n_{k} y(\mathbf{n}) \sin \left(\mathbf{n} \cdot \lambda^{0}\right)\right] \\
& +\frac{2}{N_{k} \cdot N^{2}}\left[\sum_{\mathbf{n}=\mathbf{1}}^{N} y(\mathbf{n}) \sin \left(\mathbf{n} \cdot \lambda^{0}\right)\right] \cdot\left[\sum_{\mathbf{n}=\mathbf{1}}^{N} n_{k} y(\mathbf{n}) \cos \left(\mathbf{n} \cdot \lambda^{0}\right)\right] \\
= & -2\left[\frac{1}{N} \sum_{\mathbf{n}=\mathbf{1}}^{N}\left(A^{0} \cos \left(\mathbf{n} \cdot \lambda^{0}\right)+B^{0} \sin \left(\mathbf{n} \cdot \lambda^{0}\right)+X(\mathbf{n})\right) \cos \left(\mathbf{n} \cdot \lambda^{0}\right)\right] \\
& \times\left[\frac{1}{N_{k} N} \sum_{\mathbf{n}=\mathbf{1}}^{N} n_{k}\left(A^{0} \cos \left(\mathbf{n} \cdot \lambda^{0}\right)+B^{0} \sin \left(\mathbf{n} \cdot \lambda^{0}\right)+X(\mathbf{n})\right) \sin \left(\mathbf{n} \cdot \lambda^{0}\right)\right] \\
& +2\left[\frac{1}{N} \sum_{\mathbf{n}=\mathbf{1}}^{N}\left(A^{0} \cos \left(\mathbf{n} \cdot \lambda^{0}\right)+B^{0} \sin \left(\mathbf{n} \cdot \lambda^{0}\right)+X(\mathbf{n})\right) \sin \left(\mathbf{n} \cdot \lambda^{0}\right)\right] \\
& \times\left[\frac{1}{N_{k} N} \sum_{\mathbf{n}=\mathbf{1}}^{N} n_{k}\left(A^{0} \cos \left(\mathbf{n} \cdot \lambda^{0}\right)+B^{0} \sin \left(\mathbf{n} \cdot \lambda^{0}\right)+X(\mathbf{n})\right) \cos \left(\mathbf{n} \cdot \lambda^{0}\right)\right] \\
\longrightarrow & -2\left(\frac{1}{2} A^{0}\right) \times\left(\frac{1}{4} B^{0}\right)+2\left(\frac{1}{2} B^{0}\right) \times\left(\frac{1}{4} A^{0}\right)=0, \quad k=1,2,3 .
\end{aligned}
$$

Thus $(1 / N) \mathbf{I}^{\prime}\left(\lambda^{0}\right) \mathbf{D}_{1} \rightarrow 0$, almost surely. In a similar way, it can be shown that $\left[(1 / N) \mathbf{D}_{1} \mathbf{I}^{\prime \prime}(\bar{\lambda}) \mathbf{D}_{1}\right] \rightarrow(1 / 24)\left(A^{0^{2}}+B^{0^{2}}\right) \mathbf{I}$, therefore the result follows.

Proof of Theorem 5 Note that

$$
\tilde{A}=\frac{2}{N} \sum_{\mathbf{n}=1}^{N}\left(A^{0} \cos \left(\mathbf{n} \cdot \lambda^{0}\right)+B^{0} \sin \left(\mathbf{n} \cdot \lambda^{0}\right)+X(\mathbf{n})\right) \cos (\mathbf{n} \cdot \tilde{\lambda}) .
$$

Using Lemma 2, it follows that as $N_{(1)} \rightarrow \infty$

$$
\frac{2}{N} \sum_{\mathbf{n}=1}^{N} X(\mathbf{n}) \cos (\mathbf{n} \cdot \tilde{\lambda}) \longrightarrow 0 \quad \text { a.s. }
$$

Expanding $\cos (\mathbf{n} \cdot \tilde{\lambda})$ around the point $\mathbf{n} \cdot \lambda^{0}$ by Taylor series and using Lemma 5 , it follows that as $N_{(1)} \rightarrow \infty$

$$
\frac{2}{N} \sum_{\mathbf{n}=\mathbf{1}}^{N} A^{0} \cos \left(\mathbf{n} \cdot \lambda^{0}\right) \cos (\mathbf{n} \cdot \tilde{\lambda}) \longrightarrow A^{0}, \quad \text { a.s. }
$$


and

$$
\frac{2}{N} \sum_{\mathbf{n}=\mathbf{1}}^{N} A^{0} \sin \left(\mathbf{n} \cdot \lambda^{0}\right) \cos (\mathbf{n} \cdot \tilde{\lambda}) \longrightarrow 0, \quad \text { a.s. }
$$

Similarly, it can be shown that $\tilde{B} \rightarrow B^{0}$ almost surely, therefore the result follows.

Proof of Theorem 6 Observe that

$$
\begin{aligned}
\frac{1}{N} Q_{N}(\boldsymbol{\theta})= & \frac{1}{N} \sum_{\mathbf{n}=\mathbf{1}}^{N} y(\mathbf{n})^{2}-\frac{2}{N} \sum_{\mathbf{n}=\mathbf{1}}^{N} y(\mathbf{n})(A \cos (\mathbf{n} \cdot \lambda)+B \sin (\mathbf{n} \cdot \boldsymbol{\lambda})) \\
& +\frac{1}{N} \sum_{\mathbf{n}=\mathbf{1}}^{N}(A \cos (\mathbf{n} \cdot \boldsymbol{\lambda})+B \sin (\mathbf{n} \cdot \boldsymbol{\lambda}))^{2} \\
= & \frac{1}{N} \sum_{\mathbf{n}=\mathbf{1}}^{N} y(\mathbf{n})^{2}-\frac{1}{N} J_{N}(\boldsymbol{\theta})+O\left(\frac{1}{N}\right),
\end{aligned}
$$

where

$$
\frac{1}{N} J_{N}(\boldsymbol{\theta})=\frac{2}{N} \sum_{\mathbf{n}=\mathbf{1}}^{N} y(\mathbf{n})(A \cos (\mathbf{n} \cdot \lambda)+B \sin (\mathbf{n} \cdot \lambda))-\frac{1}{2}\left(A^{2}+B^{2}\right) .
$$

Let us denote

$$
\frac{1}{N} J_{N}^{\prime}(\boldsymbol{\theta})=\left(\frac{1}{N} \frac{\partial J_{N}(\boldsymbol{\theta})}{\partial A}, \frac{1}{N} \frac{\partial J_{N}(\boldsymbol{\theta})}{\partial B}, \frac{1}{N} \frac{\partial J_{N}(\boldsymbol{\theta})}{\partial \lambda_{1}}, \frac{1}{N} \frac{\partial J_{N}(\boldsymbol{\theta})}{\partial \lambda_{2}}, \frac{1}{N} \frac{\partial J_{N}(\boldsymbol{\theta})}{\partial \lambda_{3}}\right) .
$$

After some calculations, it can be seen that

$$
\begin{aligned}
\frac{1}{N} \frac{\partial J_{N}\left(\boldsymbol{\theta}^{0}\right)}{\partial A}=\frac{2}{N} \sum_{\mathbf{n}=\mathbf{1}}^{N} X(\mathbf{n}) \cos \left(\mathbf{n} \cdot \lambda^{0}\right)+O\left(\frac{1}{N}\right) & \\
\frac{1}{N} \frac{\partial J_{N}\left(\boldsymbol{\theta}^{0}\right)}{\partial B}=\frac{2}{N} \sum_{\mathbf{n}=\mathbf{1}}^{N} X(\mathbf{n}) \sin \left(\mathbf{n} \cdot \lambda^{0}\right)+O\left(\frac{1}{N}\right) & \\
\frac{1}{N} \frac{\partial J_{N}\left(\boldsymbol{\theta}^{0}\right)}{\partial \lambda_{k}}=\frac{2}{N} \sum_{\mathbf{n}=\mathbf{1}}^{N} n_{k} X(\mathbf{n})\left(-A^{0} \sin \left(\mathbf{n} \cdot \lambda^{0}\right)+B^{0} \cos \left(\mathbf{n} \cdot \lambda^{0}\right)\right)+O\left(\frac{1}{N_{k}}\right), & \text { for } k=1,2,3 .
\end{aligned}
$$

Therefore,

$$
Q_{N}^{\prime}\left(\boldsymbol{\theta}^{0}\right)=-J_{N}^{\prime}\left(\boldsymbol{\theta}^{0}\right)+\left[\begin{array}{c}
O(1) \\
O(1) \\
O\left(N_{2} N_{3}\right) \\
O\left(N_{1} N_{3}\right) \\
O\left(N_{1} N_{2}\right)
\end{array}\right]^{\prime} .
$$

Observe that $\boldsymbol{\theta}$ that maximizes $J_{N}(\boldsymbol{\theta})$ is the same as the ALSE of $\boldsymbol{\theta}^{0}$. Now similar to equation (8), we have the following:

$$
\left(\tilde{\boldsymbol{\theta}}-\boldsymbol{\theta}^{0}\right) \mathbf{D}^{-1}=-\left[J_{N}^{\prime}\left(\boldsymbol{\theta}^{0}\right) \mathbf{D}\right]\left[\mathbf{D} J^{\prime \prime}(\overline{\boldsymbol{\theta}}) \mathbf{D}\right]^{-1} .
$$


Therefore, using equations (31) and (8), equation (32) can be written as

$$
\begin{aligned}
\left(\tilde{\boldsymbol{\theta}}-\boldsymbol{\theta}^{0}\right) \mathbf{D}^{-1} & =\left[Q_{N}^{\prime}\left(\boldsymbol{\theta}^{0}\right) \mathbf{D}+\left[\begin{array}{c}
O(1) \\
O(1) \\
O\left(N_{2} N_{3}\right) \\
O\left(N_{1} N_{3}\right) \\
O\left(N_{1} N_{2}\right)
\end{array}\right]^{\prime} \mathbf{D}\right]\left[\mathbf{D} J_{N}^{\prime \prime}(\overline{\boldsymbol{\theta}}) \mathbf{D}\right]^{-1} \\
& =\left[-\left(\hat{\boldsymbol{\theta}}-\boldsymbol{\theta}^{0}\right) \mathbf{D}^{-1}\left[\mathbf{D} Q_{N}^{\prime \prime}(\overline{\boldsymbol{\theta}}) \mathbf{D}\right]+\left[\begin{array}{c}
O(1) \\
O(1) \\
O\left(N_{2} N_{3}\right) \\
O\left(N_{1} N_{3}\right) \\
O\left(N_{1} N_{2}\right)
\end{array}\right]^{\prime}\right]\left[\mathbf{D} J_{N}^{\prime \prime}(\overline{\boldsymbol{\theta}}) \mathbf{D}\right]^{-1} .
\end{aligned}
$$

As

$$
\begin{aligned}
\lim _{N_{(1)} \rightarrow \infty}\left[\mathbf{D} J_{N}^{\prime \prime}(\overline{\boldsymbol{\theta}}) \mathbf{D}\right] & =\lim _{N_{(1)} \rightarrow \infty}\left[\mathbf{D} J_{N}^{\prime \prime}\left(\boldsymbol{\theta}^{0}\right) \mathbf{D}\right]=-\lim _{N_{(1)} \rightarrow \infty}\left[\mathbf{D} Q_{N}^{\prime \prime}(\overline{\boldsymbol{\theta}}) \mathbf{D}\right] \\
& =-\lim _{N_{(1)} \rightarrow \infty}\left[\mathbf{D} Q_{N}^{\prime \prime}\left(\boldsymbol{\theta}^{0}\right) \mathbf{D}\right]=-\boldsymbol{\Sigma}
\end{aligned}
$$

and

$$
\lim _{N_{(1)} \rightarrow \infty}\left[\begin{array}{c}
O(1) \\
O(1) \\
O\left(N_{2} N_{3}\right) \\
O\left(N_{1} N_{3}\right) \\
O\left(N_{1} N_{2}\right)
\end{array}\right]^{\prime} \mathbf{D}=\mathbf{0},
$$

it follows that the $\left(\tilde{\boldsymbol{\theta}}-\boldsymbol{\theta}^{0}\right) \mathbf{D}^{-1}$ and $\left(\hat{\boldsymbol{\theta}}-\boldsymbol{\theta}^{0}\right) \mathbf{D}^{-1}$ have the same asymptotic distributions.

\section{Appendix C}

\section{Modification of Lemma 2}

Lemma $2^{*}$ Let $\left\{X(\mathbf{n}) ; n_{1}, \ldots, n_{m}\right\}$ be a stationary random field satisfying Assumption 3, then as $N_{(1)} \rightarrow \infty$

$$
\sup _{\lambda} \frac{1}{N}\left|\sum_{\mathbf{n}=\mathbf{1}}^{N} X(\mathbf{n}) \mathrm{e}^{i(\mathbf{n} \cdot \lambda)}\right| \longrightarrow 0 \quad \text { a.s }
$$

Proof of Lemma 2* Proof of Lemma 2* goes along the same line as that of Lemma 2 except at the last stage, where we need to prove

$$
\frac{1}{N^{5}} \sup _{\lambda} \sup _{R}\left|\sum_{n_{1}=1}^{R_{1}} \cdots \sum_{n_{m}=1}^{R_{m}} X(\mathbf{n}) \mathrm{e}^{i(\mathbf{n} \cdot \lambda)}-\sum_{n_{1}=1}^{N_{1}^{5}} \cdots \sum_{n_{m}=1}^{N_{m}^{5}} X(\mathbf{n}) \mathrm{e}^{i(\mathbf{n} \cdot \lambda)}\right| \longrightarrow 0 \quad \text { a.s. }
$$


Here, $R$ denotes the range of $R_{1}, \ldots, R_{m}$ and $N_{1}^{5}<R_{1} \leq\left(N_{1}+1\right)^{5}, \ldots, N_{m}^{5}<R_{m} \leq\left(N_{m}+\right.$ $1)^{5}$. Observe that

$$
\begin{aligned}
& \frac{1}{N^{5}} \sup _{\lambda} \sup _{R}\left|\sum_{n_{1}=1}^{R_{1}} \cdots \sum_{n_{m}=1}^{R_{m}} X(\mathbf{n}) \mathrm{e}^{i(\mathbf{n} \cdot \lambda)}-\sum_{n_{1}=1}^{N_{1}^{5}} \cdots \sum_{n_{m}=1}^{N_{m}^{5}} X(\mathbf{n}) \mathrm{e}^{i(\mathbf{n} \cdot \lambda)}\right| \\
& \leq \frac{1}{N^{5}} \sup _{\lambda} \sup _{R_{m}}\left|\sum_{n_{1}=1}^{N_{1}^{5}} \cdots \sum_{n_{m-1}=1}^{N_{m-1}^{5}} \sum_{n_{m}=N_{m}^{5}+1}^{R_{m}} X(\mathbf{n}) \mathrm{e}^{i(\mathbf{n} \cdot \lambda)}\right|\left[\left(\begin{array}{c}
m \\
1
\end{array}\right) \text { additive terms like this }\right] \\
& +\frac{1}{N^{5}} \sup _{\lambda} \sup _{R_{m-1} R_{m}}\left|\sum_{n_{1}=1}^{N_{1}^{5}} \cdots \sum_{n_{m-2}=1}^{N_{m-2}^{5}} \sum_{n_{m-1}=N_{m-1}^{5}+1}^{R_{m-1}} \sum_{n_{m}=N_{m}^{5}+1}^{R_{m}} X(\mathbf{n}) \mathrm{e}^{i(\mathbf{n} \cdot \lambda)}\right| \\
& \times\left[\left(\begin{array}{c}
m \\
2
\end{array}\right) \text { additive terms like this }\right] \\
& +\cdots+\frac{1}{N^{5}} \sup _{\lambda} \sup _{R_{2} \ldots R_{m}}\left|\sum_{n_{1}=1}^{N_{1}^{5}} \sum_{n_{2}=N_{2}^{5}+1}^{R_{2}} \cdots \sum_{n_{m}=N_{m}^{5}+1}^{R_{m}} X(\mathbf{n}) \mathrm{e}^{i(\mathbf{n} \cdot \lambda)}\right| \\
& \times\left[\left(\begin{array}{c}
m \\
m-1
\end{array}\right) \text { additive terms like this }\right]+\frac{1}{N^{5}} \sup _{\lambda}\left|\sum_{n_{1}=N_{1}^{5}+1}^{\left(N_{1}+1\right)^{5}} \cdots \sum_{n_{m}=N_{m}^{5}+1}^{\left(N_{m}+1\right)^{5}} X(\mathbf{n}) \mathrm{e}^{i(\mathbf{n} \cdot \lambda)}\right| \\
& \leq O\left(\left(N_{1} \ldots N_{m-1}\right)^{-(5 / 4)} N_{m}^{-2}\right)+O\left(\left(N_{1} \ldots N_{m-2}\right)^{-(5 / 4)}\left(N_{m-1} N_{m}\right)^{-2}\right)+\cdots+O\left(N^{-2}\right) \text {. }
\end{aligned}
$$

The last inequality follows sequentially from 3-D results. Therefore, all the terms converge to zero and that proves the lemma.

Proof of Theorem 7 Using Lemma 1 and Lemma 2*, the result follows using the similar approach as Theorem 1 .

Proof of Theorem 8 The proof follows exactly the same way as Theorem 2.

\section{References}

[1] Hua, Y., 1991, Matrix pencil and multidimensional sinusoids. Proceedings of the Seventh Workshop on Multidimensional Signal Processing, Lake Placid, New York, IEEE Publications, 2.1.

[2] Liang, X., Tao, R., Zhou, S. and Wang, Y., 2001, Multidimensional real aperture radar imaging. Proceedings of CIE International Conference on Radar, pp. 675-678.

[3] Chylla, R.A. and Markley, J.L., 1995, Theory and applications of the maximum likelihood principle to NMR parameter estimation of multidimensional NMR data. Journal of Bimolecular NMR, 5, 245-258.

[4] Kundu, D., 1997, Asymptotic theory of the least squares estimators of sinusoidal signals. Statistics, 30(3), 221-238.

[5] Fisher, R.A., 1929, Tests of significance in harmonic analysis. Proceedings of the Royal Society of London, Series A, 125, 54-59.

[6] Hannan, E.J., 1971, Non-linear time series regression. Journal of the Applied Probability, 8, 767-780.

[7] Hannan, E.J., 1973, The estimation of frequency. Journal of the Applied Probability, 10, 510-519.

[8] Walker, A.M., 1971, On the estimation of a harmonic components in a time series with stationary residuals. Biometrika, 58, 21-36.

[9] Kundu, D., 1993, Asymptotic theory of least squares estimators of a particular non-linear regression model. Statistics and Probability Letters, 18(1), 13-17.

[10] Rice, J.A. and Rosenblatt, M., 1988, On frequency estimation. Biometrika, 75(3), 477-484.

[11] Stoica, P., 1993, List of references on spectral line analysis. Signal Processing, 31, 329-340.

[12] Barbieri, M.M. and Barone, P., 1992, A two dimensional Prony's method for Spectral estimation. IEEE Transactions on Signal Processing, 40(11), 2747-2756. 
[13] Cabrera, S.D. and Bose, N.K., 1993, Prony methods for two dimensional complex signals modeling. In: S.G. Tzafestas, Applied Control, Chap. 15 (Deker) pp. 401-411.

[14] Chun, J. and Bose, N.K., 1995, Parameter estimation via signal selectivity of signal subspaces (PESS) and its applications to 2-D wave number estimation. Digital Signal Processing, 5, 58-76.

[15] Hua, Y., 1992, Estimating two dimensional frequencies by matrix enhancement pencil. IEEE Transactions on Signal Processing, 40(9), 2267-2280.

[16] Kundu, D. and Gupta, R.D., 1998, Asymptotic properties of the least squares estimators of a two dimensional textures model. Metrika, 48, 83-97.

[17] Lang, S.W. and McClellan, J.H., 1982, The extension of Pisarenko method to multiple sinusoids. Proc. ICASSP82, Paris, France, pp. 125-128.

[18] Kundu, D. and Mitra, A., 1996, Asymptotic properties of the least squares estimators of 2-D exponential signals. Multidimensional Systems and Signal Processing, 7(3), 135-150.

[19] Nandi, S. and Kundu, D., 1999, Least-squares estimators in a stationary random field. Journal of the Indian Institute of Science, $\mathbf{7 9}, 75-88$.

[20] Kundu, D. and Nandi, S., 2001, On asymptotic properties of a two dimensional frequency estimators. Communications in Statistics - Theory and Methods, 30(8-9), 1561-1577.

[21] Kundu, D. and Nandi, S., 2003, Determination of discrete spectrum in a random field. Statistica Neerlandica, 57(2), 258-283.

[22] Rao, C.R. Zhao, L.C., and Zhou, B., 1994, Maximum likelihood estimators of 2-D superimposed exponential signals. IEEE Transactions on Acoustics, Speech and Signal Processing, 42(7) 1795-1802,

[23] Mitra, A. and Stoica, P., 2002, The asymptotic Cramer-Rao bound for 2-D superimposed exponential signals. Multidimensional Systems and Signal Processing, 13, 317-331.

[24] Mangulis, V., 1965, Handbook of Series for Scientists and Engineers (New York: Academic Press).

[25] Fuller, W.A., 1976, Introduction to Statistical Time Series (New York: John Wiley and Sons).

[26] Wu, C.F.J., 1981, Asymptotic theory of non linear least squares estimation. Annals of Statistics, 9, 501-513. 
